Proceedings of the 1996 IEEE

International Conference on Robotics and Automation

Minneapolis, Minnesota - April 1996

\title{
On Force and Form Closure For Multiple Finger Grasps
}

\author{
Elon Rimon \\ Dept. of Mechanical Engineering \\ Technion, Israel Inst. of Technology \\ Haifa, ISRAEL
}

\author{
Joel Burdick \\ Dept. of Mechanical Engineering \\ California Inst. of Technology \\ Pasadena, CA 91125
}

\begin{abstract}
This paper considers the relationship between force and form closure. Based on a recently developed mobility theory, we give precise definitions for $1^{\text {st }}$ and $2^{\text {nd }}$ order form closure for frictioniess grasps. We also introduce the new concept of $2^{\text {nd }}$ order force closure. We show for the case of frictionless contacts, a grasp is $1^{\text {st }}$ order force closure if and only if it is $1^{\text {st }}$ order form closure. We further show that a grasp is $2^{\text {nd }}$ order force closure if and only if it is also $2^{\text {nd }}$ order form closure.
\end{abstract}

\section{Introduction}

This paper considers force closure, form closure, and the relationship between the two. In the first part of this paper, we consider these issues in the case of frictionless contacts. Force closure is a well established theory, and researchers have converged on a consistent set of definitions and mathematics for its use in the analysis of grasping and fixturing. On the other hand, the concept of form closure is less well defined in the robotics literature, as different authors have used this term to describe similar, but slightly different, concepts. One of the goals of this paper is to introduce precise notions of form closure and relate them to force closure. We give precise definitions of $1^{\text {st }}$ and $2^{\text {nd }}$ order form closure. Further, we show that $1^{\text {st }}$ order form closure implies force closure (which we term $1^{\text {st }}$ order force closure), and vice versa. We also introduce a novel $2^{\text {nd }}$ order force closure definition, and show that our $2^{\text {nd }}$-order form closure definition is dual to $2^{\text {nd }}$-order force closure.

Our analysis and discussion is based on a recent mobility theory which has been developed by the authors $[10,11,12,13]$. This theory determines the mobility of a smooth object, $\mathcal{B}$, held in frictionless contact by smooth rigid and stationary finger bodies or fixtures $\mathcal{A}_{1}, \ldots, \mathcal{A}_{k}$. Our mobility analysis (which is reviewed in Section 3) is formulated in configuration space (cspace).

\section{Relation to Previous Work}

The history of force/form closure analysis dates back to the work of Reuleaux (1876) [9] and Somoff (1900)
[15]. More recent analysis dates to the work of Lakshminarayana [3]. Numerous investigators have provided various equivalent definitions of force closure. However, all of these definitions are based on what we term $1^{\text {st }}$ order notions of velocity and force. Our definition of $2^{\text {nd }}$ order force closure appears to be new.

The concept of form closure has not been precisely defined in the literature. Mirtich and Canny [5] consider a grasp to be form closure when $\mathcal{B}$ 's configuration, which is represented by a point in configuration space, is surrounded by c-space obstacles. However, they give no analytical or computational procedure whereby one could determine if a grasp is form closure. Their notion coincides with our concept of immobility. However, we give more precise definitions of $1^{\text {st }}$ and $2^{\text {nd }}$ order immobility (or form closure) which can be determined by calculating $1^{s t}$ and $2^{\text {nd }}$ order mobility indices. Others (for example [16]) use a notion of form closure which is similar to our notion of $1^{\text {st }}$ order form closure. Other investigators have used Screw Theory (a first order theory) to study the relative motions of bodies in contact [8]. Our notion of $1^{\text {st }}$ order mobility analysis gives results that are equivalent to Screw Theory.

However, traditional $1^{\text {st }}$-order theories do not account for higher order geometrical effects. For example, in Fig. 1 a smooth triangular object is grasped by 3 point "fingers" or fixtures. First-order theories would indicate that the object can instantaneously rotate about the point of concurrency of the contact normals. However, if the fingers are rigidly immobile, the triangular object is in fact completely immobilized. First-order theories are inadequate because the relative mobility of an object in contact with finger bodies is not an infinitesimal notion, but a local one. This deficiency motivated our development of a novel $2^{\text {nd }}$ order mobility theory, which includes the curvatures of the contacting surfaces in the analysis of mobility. While examples show that $1^{\text {st }}$ order effects alone are insufficient to correctly characterize mobility, the combination of $1^{\text {st }}$ and $2^{\text {nd }}$ effects does suffice to completely characterize the mobility of generic grasps.

Our $2^{\text {nd }}$ order mobility theory includes the effects of 
curvature, or "higher order" kinematics in mobility analysis. Prior researchers have also included curvature or $2^{\text {nd }}$ order effects in the analysis of grasp stability. Howard and Kumar [2] have included finger and object curvatures in the calculation of the stiffness matrix for compliant grasps. Their compliance model, which is based on a simple linear spring idealization, can be used to assess the stability of a given grasp. In comparison, Rimon and Burdick [11] have shown that kinematic immobility automatically implies dynamic stability for a large class of nonlinear compliance models. Trinkle et. al [18] have also considered $2^{\text {nd }}$ terms in the stability analysis of frictionless grasps of polygonal objects. While Ref.s [2] and [18] both give means to determine the stability of a given grasp (under the assumptions of their models), neither work addresses the more general issue of how to develop a unifying viewpoint on the issues of force and form closure.

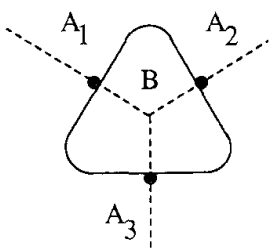

Figure 1: A 3-finger immobilizing grasp

Although the main goal of this paper is to consider the more fundamental issue of the definitions of force/form closure, it is worth noting that the concepts of force and form closure have been used to analyze grasping [7, 16], fixturing [6], and whole arm manipulation [17]. One fundamental application of the force/form closure concept is the issue of the number of frictionless contacts that are required to immobilize an object. Reuleaux [9] found that at least 4 frictionless point contacts are required to immobilize 2-dimensional (2D) objects. Somoff [15] found that at least 7 frictionless contact points are required to immobilize 3-dimensional (3D) objects. Much later, Markenscoff et. al (1990) [4] established that 4 contact points suffice to immobilize generic 2D objects, and 7 suffice to immobilize generic 3D objects. Czyzowicz et. al (1991) [1] have shown that generic 2D and $3 \mathrm{D}$ polygonal objects could be immobilized respectively by 3 and 4 frictionless point contacts. More recently, Rimon and Burdick [14] have shown that any $2 \mathrm{D}$ polygonal or smooth object can immobilized with 3 convex fingers (some fingers might have to be flat). They were also able to overcome the limitation of the analysis in [1] to polygons without parallel edges. Further, they have shown that if the finger curvature can be chosen, then it is possible to immobilize planar smooth and polygonal objects with 2

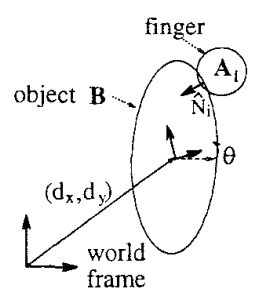

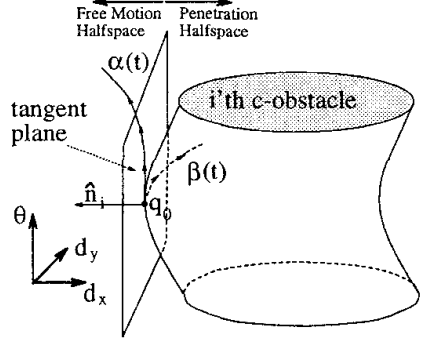

(b)
Figure 2: Schematic view of c-space obstacle.

frictionless finger contacts.

\section{Rigid Body Mobility Analysis}

The essential components of our mobility theory are now reviewed, as these concepts are the basis for our discussions. We study the mobility of an object $\mathcal{B}$ held in point contact by $k$ stationary and frictionless finger bodies $\mathcal{A}_{1}, \ldots, \mathcal{A}_{k}$. The analysis is formulated in $\mathcal{B}$ 's configuration space. The object $\mathcal{B}$ is described in its own nominal space $\mathcal{R}=\mathbb{R}^{n}(n=2$ or 3$)$, in terms of a body fixed reference frame in $\mathcal{R}, F_{\mathcal{B}}$. Points in $\mathcal{R}$ are denoted $r$. Points in the physical space, $\mathcal{E}$, are denoted $x$. Every rigid placement of $\mathcal{B}$ in $\mathcal{E}$ is described by the following rigid body transformation:

$$
x=X(r,(d, R)) \triangleq R r+d \quad r \in \mathcal{R}, x \in \mathcal{E} .
$$

The c-space of $\mathcal{B}$ is parametrized in terms of hybrid coordinates by $q=(d, \theta)$, where $d \in \mathbb{R}^{3}$ directly parametrizes translation and $\theta \in \mathbb{I}^{3}$ parametrizes $S O(3)$ via the usual exponential map, where $\widehat{\theta}=$ $\theta /\|\theta\|$ is the axis of rotation and $\|\theta\|$ is the angle of rotation. Thus $\mathcal{B}$ 's c-space is parametrized by $\mathbb{R}^{m}$, where $m=\frac{1}{2} n(n+1)(m=3$ or 6$)$.

The fingers are represented as c-space obstacles (or $c$-obstacles). For example, in Fig. $2(a) \mathcal{B}$ is contacted by a single finger $\mathcal{A}_{i}$. $\mathcal{B}$ 's c-space is $q=\left(d_{x}, d_{y}, \theta\right)$, and the c-obstacle due to $\mathcal{A}_{i}$ (shown schematically in Fig. 2(b)) is the set of all configurations where $\mathcal{B}$ intersects the stationary "obstacle" $\mathcal{A}_{i}$. Thus, if $q_{0}$ is $\mathcal{B}^{\prime}$ 's contact configuration, $q_{0}$ lies on the $i^{t h} \mathrm{c}^{-}$ obstacle boundary, which is denoted $\mathcal{S}_{i}$. When $\mathcal{B}$ is contacted by $k$ fingers, $q_{0}$ lies on the intersection of $\mathcal{S}_{i}$ for $i=1, \ldots, k$.

\section{$3.11^{\text {st }}$ and $2^{\text {nd }}$ order free motions}

The free motions of $\mathcal{B}$ are those local motions of $\mathcal{B}$ along which it either breaks away from or roll-slides ${ }^{1}$

\footnotetext{
${ }^{1} \mathrm{By}$ a "roll-slide" motion we mean a general displacement between two bodies which maintains surface contact.
} 
on the surface of the finger bodies. In c-space, the free motions of $\mathcal{B}$ at $q_{0}$ are the c-space paths that emanate from $q_{0}$ and locally lie in the freespace, which is the complement of the c-obstacle interiors. The first-order properties of the free motion paths and the c-obstacle boundaries (i.e. tangents and tangent hyperplanes) determine what we call the $1^{\text {st }}$ order mobility of $\mathcal{B}$. This notion can be equated to other well known first-order theories, such as Screw Theory [8]. We cast these notions in a c-space framework, as this interpretation is the basis for the consideration of higher order aspects of mobility. In the following, $\hat{n}_{i}\left(q_{0}\right)$ denotes the outward pointing unit normal to $\mathcal{S}_{i}$ at $q_{0}$ (Fig. $2(\mathrm{~b})$ ).

Definition 1 : The $1^{\text {st }}$ order free motions of $\mathcal{B}$ at $q_{0}$ is the halfspace of tangent vectors $\dot{q} \in T_{q_{0}} \mathbb{R}^{m}$ satisfying $M_{i}^{1}\left(q_{0}\right) \triangleq\left\{\dot{q} \in T_{q_{0}} \mathbb{R}^{m}: \hat{\mathrm{n}}_{i}\left(q_{0}\right) \cdot \dot{q} \geq 0\right\}$. The halfspace's boundary, $T_{q_{0}} \mathcal{S}_{i}=\left\{\dot{q}: \hat{\mathrm{n}}_{i}\left(q_{0}\right) \cdot \dot{q}=0\right\}$, is called the set of $1^{\text {st }}$ order roll-slide motions. Its interior, $\left\{\dot{q}: \hat{\mathrm{n}}_{i}\left(q_{0}\right) \cdot \dot{q}>0\right\}$, is termed the set of $\mathbf{1}^{\text {st }}$ order escape motions. For $k$ fingers, the set of $1^{\text {st }}$ order free motions is: $M_{1, \ldots, k}^{1}\left(q_{0}\right) \triangleq \bigcap_{i=1}^{k} M_{i}^{1}\left(q_{0}\right)$.

In other words, $\mathcal{B}$ is increasing its distance from $\mathcal{A}_{i}$ to first order along $1^{\text {st }}$ order escape motions, which implies that it locally breaks away from $\mathcal{A}_{i}$. To first order, $\mathcal{B}$ maintains zero distance from $\mathcal{A}_{i}$ along $1^{\text {st }}$ order roll-slide motions, and it is not possible to determine from $1^{\text {st }}$ order considerations if $\mathcal{B}$ locally breaks away or penetrates $\mathcal{A}_{i}$. For example, the c-space curves $\alpha(t)$ and $\beta(t)$ in Fig. 2 are equivalent to first order, yet $\alpha(t)$ locally lies in the freespace, while $\beta(t)$ does not. It can be shown that all the free motions of $\mathcal{B}$ at an equilibrium grasp are necessarily roll-slide to $1^{\text {st }}$ order [12]. Hence, $\mathcal{B}$ 's mobility depends on the second order properties of its local motions.

The second-order geometry of the free-motion curves and the c-obstacle boundaries is determined by their curvature and curvature form, respectively. The curvature form of $\mathcal{S}_{i}$ at $q_{0} \in \mathcal{S}_{i}$ is denoted $\kappa_{i}\left(q_{0}, \dot{q}\right)$, where $\kappa_{i}\left(q_{0}, \dot{q}\right)=\dot{q}^{T}\left[D \hat{\mathbf{n}}_{i}\left(q_{0}\right)\right] \dot{q}$ for $\dot{q} \in T_{q_{0}} \mathcal{S}_{i}$. The matrix $D \hat{\mathrm{n}}_{i}\left(q_{0}\right)=D^{2} d_{i}\left(q_{0}\right)$ encodes the curvature of the c-obstacle surface at $q_{0}$. In Ref. [13] we give a closed-form formula for the curvature form in terms of the surface curvatures and location of the contacting bodies. We show in Ref. [12] that the free motion curves are determined to $2^{\text {nd }}$-order by their velocity and acceleration at $q_{0}$.

Definition 2: The $2^{\text {nd }}$ order free motions of $\mathcal{B}$ at $q_{0}$ is the subset of $(\dot{q}, \ddot{q})$ satisfying
$M_{i}^{2}\left(q_{0}\right) \triangleq\left\{(\dot{q}, \ddot{q}): \hat{\mathrm{n}}_{i}\left(q_{0}\right) \cdot \dot{q}=0\right.$ and $\dot{q}^{T}\left[D \hat{\mathrm{n}}_{i}\left(q_{0}\right)\right] \dot{q}+$ $\left.\hat{\mathrm{n}}_{i}\left(q_{0}\right) \cdot \ddot{q} \geq 0\right\}$. Pairs $(\dot{q}, \ddot{q})$ that satisfy $\hat{\mathrm{n}}_{i}\left(q_{0}\right) \cdot \dot{q}=0$ and $\dot{q}^{T}\left[D \hat{\mathrm{n}}_{i}\left(q_{0}\right)\right] \dot{q}+\hat{\mathrm{n}}_{i}\left(q_{0}\right) \cdot \ddot{q}=0$ are called $2^{\text {nd }}$ order roll-slide motions, and the other pairs in $M_{i}^{2}\left(q_{0}\right)$ are termed $\mathbf{2}^{\text {nd }}$ order escape motions. For $k$ fingers, $M_{1, \ldots, k}^{2}\left(q_{0}\right) \triangleq \bigcap_{i=1}^{k} M_{i}^{2}\left(q_{0}\right)$.

We show in Ref. [12] that if $(\dot{q}, \ddot{q}) \in M_{i}^{2}\left(q_{0}\right)$ is a $2^{\text {nd }}$ order escape motion, its corresponding c-space path, $\alpha(t)$ with $\alpha(0)=q_{0}, \dot{\alpha}(0)=\dot{q}, \ddot{\alpha}(0)=\ddot{q}$, locally lies in the freespace for $t \geq 0$.

\section{$3.2 \quad 1^{\text {st }}$ and $2^{\text {nd }}$ Order Mobility Indices}

Mobility indices are coordinate invariant integervalued functions that measure the mobility, or effective number of degrees of freedom, of $\mathcal{B}$ when it is held in an equilibrium-grasp configuration $q_{0}$. At an equilibrium grasp the net wrench on $\mathcal{B}$ must be zero. In c-space, the wrench due to a normal contact force applied by $\mathcal{A}_{i}$ on $\mathcal{B}$ is represented as a positive multiple of the outward pointing finger c-obstacle normals $\hat{\mathrm{n}}_{i}\left(q_{0}\right)[12]$. The equilibrium condition in c-space is thus characterized by the requirement that the origin of $T_{q_{0}} \mathbb{R}^{m}$ be in the convex hull of the finger cobstacles' normals. That is, there must exist scalars $\lambda_{1}, \ldots, \lambda_{k}$ such that

$$
\begin{aligned}
0= & \lambda_{1} \hat{\mathbf{n}}_{1}\left(q_{0}\right)+\cdots+\lambda_{\mathbf{k}} \hat{\mathbf{n}}_{k}\left(q_{0}\right), \\
& \lambda_{i} \geq 0 \text { and } \sum_{i=1}^{k} \lambda_{i}=1 .
\end{aligned}
$$

We assume that each $\hat{\mathrm{n}}_{i}\left(q_{0}\right)$ in (2) is essential for the grasp, meaning that the origin cannot be positively spanned with any subcollection of $\left\{\hat{n}_{1}\left(q_{0}\right), \ldots, \hat{n}_{k}\left(q_{0}\right)\right\}$ [10]. Grasps are generically essential in the planar case for $k=2,3,4$ and in the spatial case for $k=$ $2, \ldots, 7$. It can be shown that at a $k$-fingered equilibrium grasp, $M_{1, \ldots, k}^{1}\left(q_{0}\right)$ forms a subspace [12]. This subspace consists of $1^{\text {st }}$ order motions which are rollslide with respect to each of the fingers. For essential grasps, the dimension of $M_{1, \ldots, k}^{1}\left(q_{0}\right)$ is $(m-k+1)$ ( $m=3$ or 6$)$. The $1^{\text {st }}$ order mobility index of an equilibrium grasp, denoted $m_{q_{0}}^{1}$, is defined as the dimension of this subspace. Thus $m_{q_{0}}^{1}=(m-k+1)$ in the essential case. $m_{q_{0}}^{1}$ is shown in Ref. [12] to be coordinate invariant. A key fact is that $m_{q_{0}}^{1}$ is identical for all $k$-fingered grasps. Thus, any first order theory, such as screw theory, will be unable to distinguish between alternative equilibrium grasps which have the same number of fingers. This lack of discriminating power is remedied with our $2^{\text {nd }}$ order index.

Consider the $\lambda_{i}$ 's in the equilibrium condition (2). While the individual c-obstacle curvature forms are in general not coordinate invariant, it is shown in Ref. 
[10] that their weighted sum, called the relative $c$ space curvature, has a coordinate invariant structure which characterizes the $2^{\text {nd }}$ order mobility of $\mathcal{B}$.

Definition $3[10]$ : Let $\lambda_{1}, \ldots, \lambda_{k}$ be the coefficients of the equilibrium equation (2). The c-space relative curvature form for the equilibrium grasp is the quadratic form: $\kappa_{\mathrm{rel}}\left(q_{0}, \dot{q}\right) \triangleq \sum_{i=1}^{k} \lambda_{i} \kappa_{i}\left(q_{0}, \dot{q}\right)$, such that $\dot{q} \in M_{1, \ldots, k}^{1}\left(q_{0}\right)$.

The $2^{\text {nd }}$ order mobility index of an equilibrium grasp configuration, denoted $m_{q_{0}}^{2}$, is the number of non-negative eigenvalues of the c-space relative curvature, $\sum_{i=1}^{k} \lambda_{i}\left[D \hat{\mathrm{n}}_{i}\left(q_{0}\right)\right]$.

Since the relative curvature form is evaluated on the set of $1^{\text {st }}$ order free motions, $m_{q_{0}}^{1}$ is an upper bound on the values of $m_{q_{0}}^{2}$, i.e. $0 \leq m_{q_{0}}^{2} \leq m_{q_{0}}^{1}$. This inequality has an important practical interpretation: $2^{\text {nd }}$ order effects can reduce the mobility of the grasped object, as predicted by $1^{\text {st }}$ order effects. In particular, if $m_{q_{0}}^{1}=0, \mathcal{B}$ is completely immobilized, and the $2^{\text {nd }}$ order index carries no immediately useful information. The $2^{\text {nd }}$ order index is always useful for determining the mobility of planar grasps involving $k=2,3$ fingers, and in $3 \mathrm{D}$ grasps involving $k=2, \ldots, 6$ fingers. In these cases $\mathcal{B}$ is not immobilized to first order $\left(m_{q_{0}}^{1}>0\right)$, but may be immobilized to $2^{\text {nd }}$ order $\left(m_{q_{0}}^{2}=0\right)$. A key interpretation of the $2^{\text {nd }}$ order index is provided in Proposition 5.6 of Ref. [10]: if $m_{q_{0}}^{2}=0$, any local motion of $\mathcal{B}$ is either $1^{\text {st }}$ order penetration, or it is $1^{\text {st }}$ order roll-slide which is necessarily a $2^{\text {nd }}$ order penetration motion. Thus $m_{q_{0}}^{2}=0$ implies that $\mathcal{B}$ is completely immobilized, and a test of $m_{q_{0}}^{2}$ is sufficient to determine immobility.

\section{Form and Force Closure}

The dual concepts of force closure and form closure have often been discussed in the literature on multifingered grasping, fixture planning, and the kinematics of bodies in contact. Unfortunately, the distinction between these terms has at times been unclear. We revisit these issues here to point out how our work fits in the historical development of grasping and fixturing analysis and how it can be used as the basis for developing a more unified terminology.

\section{1 $1^{\text {st }}$ and $2^{\text {nd }}$ Order Form Closure}

One of the significant differences between form and force closure lies in the way in which the contact between a fixture and an object is modeled. In form closure the contact between $\mathcal{B}$ and $\mathcal{A}_{i}$ is modeled as a constraint on $\mathcal{B}$ 's allowable motions. That is, one might consider that the sole purpose of a finger or fixture is to limit the possible motions of $\mathcal{B}$. If enough constraints are placed on $\mathcal{B}$ 's motion, then $\mathcal{B}$ will be immobilized. Thus, from a practical perspective, form closure is equivalent to our notion of immobilization. From a c-space point of view, a grasp is form closure or immobilized if $\mathcal{B}$ 's configuration, $q_{0}$, is completely surrounded by c-obstacles. The concepts of $i^{\text {th }}$ order mobility and $i^{\text {th }}$ order mobility index give us a means to make the following precise characterization of form closure, which has heretofore been missing from the literature.

Definition 4 Let $\mathcal{B}$ be at configuration $q_{0}$, in contact with $k$ finger bodies. $\mathcal{B}$ is in $1^{\text {st }}$ order form closure if it is immobilized to $1^{\text {st }}$ order by the fingers i.e., if the intersection of the $1^{\text {st }}$ order free halfspaces, $M_{i}^{1}\left(q_{0}\right)$ for $i=1, \ldots, k$, contains only the origin, $\dot{q}=0$, of $T_{q_{0}} \mathbb{R}^{m}$.

$\mathcal{B}$ is held in $2^{\text {nd }}$ order form closure if it is immobilized to $2^{\text {nd }}$ order i.e., if the intersection of the $2^{\text {nd }}$ order free motion sets, $M_{i}^{2}\left(q_{0}\right)$ for $i=1, \ldots, k$, contains only the zero velocity and acceleration vectors, $\dot{q}=0$ and $\ddot{q}=0$.

For grasps whose fingers are essential, $1^{\text {st }}$ order form closure is equivalent to $m_{q_{0}}^{1}=0$, and $2^{\text {nd }}$ order form closure is equivalent to $m_{q_{0}}^{2}=0$.

Hence, the mobility indices give precise and computable tests for $1^{\text {st }}$ and $2^{\text {nd }}$ order form closure.

\section{$4.21^{\text {st }}$ and $2^{\text {nd }}$ Order Force Closure}

In the dual modeling approach of force closure, the effect of the contacting finger or fixture on $\mathcal{B}$ is modeled as a contact force. In a general frictional contact, $\mathcal{A}_{i}$ can apply a set of contact forces on $\mathcal{B}$ at a given contact point. This set reduces to a force along the surface normal for frictionless contact. Let $W_{i}$ be the collection of wrenches (i.e. force and torque) generated by applying all possible contact forces at the $i^{\text {th }}$ contact point, and let $W=W_{1}+\cdots+W_{k}$ be the collection of all wrenches which can possibly be generated by the $k$ fingers. If $W$ contains a neighborhood about the zero wrench, the grasp is said to be force closure-though the term wrench closure is more appropriate. In practice it means that any external wrench can be resisted by a proper combination of the finger forces.

We now show that frictionless $1^{\text {st }}$ order form closure is equivalent to frictionless force closure. 
Lemma 4.1 Let $\mathcal{B}$ be held in a $k$-finger frictionless equilibrium grasp, such that all the fingers apply nonzero force. Then the grasp is force closure if and only if it is $1^{\text {st }}$ order form closure.

Proof: We show that $1^{\text {st }}$ order form-closure implies force closure. For a frictionless contact $W_{i}=$ $\sigma_{i} \hat{n}_{i}\left(q_{0}\right)$ for $\sigma_{i} \geq 0$. Hence it suffices to show that the normals $\hat{n}_{i}\left(q_{0}\right)$ for $i=1, \ldots, k$ positively span a neighborhood about the origin. By definition of $1^{\text {st }}$ order form-closure, for every $\dot{q} \in T_{q_{0}} \mathbb{R}^{m}$ there exists a normal $\hat{n}_{i}\left(q_{0}\right)$ such that $\hat{n}_{i}\left(q_{0}\right) \cdot \dot{q}<0$. It follows that the $k \times m$ matrix $M$, whose rows are $\hat{n}_{i}\left(q_{0}\right)^{T}$ for $i=1, \ldots, k$, has rank $m$ (necessarily $k \geq m$ ). Otherwise there would exist a vector $\dot{q}$ such that $\hat{n}_{i}\left(q_{0}\right) \cdot \dot{q}=0$ for $i=1, \ldots, k$. But the rank of $M^{T}$ is also $m$. Hence $M^{T}$ maps open neighborhoods in its domain, $\mathbb{R}^{k}$, onto open neighborhoods in its image space, $\mathbb{R}^{m}$. From the equilibrium condition we know that $\lambda_{1} \hat{n}_{1}\left(q_{0}\right)+\cdots+\lambda_{k} \hat{n}_{k}\left(q_{0}\right)=0$. Hence $M^{T}$ maps an open neighborhood about the point $\left(\lambda_{1}, \ldots, \lambda_{k}\right)$ in $\mathbb{R}^{k}$ onto an open neighborhood about the origin in $\mathbb{R}^{m}$. Since by assumption each $\lambda_{i}$ is positive, a sufficiently small neighborhood about $\left(\lambda_{1}, \ldots, \lambda_{k}\right)$ consists of points of $\mathbb{R}^{k}$ whose coordinates are positive. Thus the normals $\hat{n}_{i}\left(q_{0}\right)$ for $i=1, \ldots, k$ positively span a neighborhood about the origin. The arguments are all reversible, hence it also follows that force closure implies $1^{\text {st }}$ order form-closure.

We have just seen that for frictionless equilibrium grasps, $1^{\text {st }}$ order form-closure is equivalent to force closure. It is therefore natural to regard force closure as $1^{\text {st }}$ order force closure, and to seek a definition of $2^{\text {nd }}$ order force closure. We propose such a definition for the case of frictionless contact, where $W_{i}=\sigma_{i} \hat{n}_{i}\left(q_{0}\right)$ for $\sigma_{i} \geq 0$. In the following definition, $U$ is the space spanned by all possible finger wrenches. We know that $U$ is a subspace $\operatorname{since} \mathcal{B}$ is in an equilibrium grasp [12]. Let $V$ be the subspace consisting of the orthogonal complement of $U$. We also use in the definition the wrench derivatives which are generated by a stationary finger $\mathcal{A}_{i}$, while $\mathcal{B}$ moves along a general roll-slide motion on the surface of $\mathcal{A}_{i}$. Since every finger wrench has the form $w_{i}=\sigma_{i} \hat{n}_{i}\left(q_{0}\right)$, its time-derivative along a c-space trajectory $q(t)$ of $\mathcal{B}$ is $\dot{w}_{i}=\dot{\sigma}_{i} \hat{n}_{i}\left(q_{0}\right)+\sigma_{i} D \hat{n}_{i}\left(q_{0}\right) \dot{q}$. Intuitively, one component of $\dot{w}_{i}$ is generated by varying the magnitude of the contact force, while the other is generated by changing the location of the contact point.

Definition 5 Let $\mathcal{B}$ be held in a $k$-finger frictionless equilibrium grasp. The grasp is $2^{\text {nd }}$ order force closure if, first, the feasible wrenches $W=W_{1}+\cdots+$
$W_{k}$ contain a neighborhood about the origin in the subspace $U$. And second, if at each $w=w_{1}+\cdots+w_{k}$ in $W$, the wrench-derivatives $\dot{w}_{i}$ for $i=1, \ldots, k$, span the orthogonal complement subspace $V$.

Note that any $1^{\text {st }}$ order force-closure grasp is automatically force-closure to $2^{\text {nd }}$ order. Let us discuss the intuition behind the definition. Every external wrench $w$ can be uniquely written as $w=u+v$, such that $u \in U$ and $v \in V$. From the definition it is clear that the $u$ component can be directly resisted by proper choice of the finger forces. The definition also requires that the fingers be able to generate a wrench derivative whose integration yields a wrench that resists the $v$ component of $w$. The following lemma gives further evidence that the definition of $2^{\text {nd }}$ order force closure captures the notion dual to $2^{\text {nd }}$ order form-closure.

Lemma 4.2 Let $\mathcal{B}$ be held at a $k$-finger frictionless equilibrium grasp, such that all the fingers are essential for the grasp. Then the grasp is $2^{\text {nd }}$ order force closure iff it is $2^{\text {nd }}$ order form closure.

Proof: We show that $2^{\text {nd }}$ order form-closure implies $2^{\text {nd }}$ order force closure. For a frictionless contact $W_{i}=\sigma_{i} \hat{n}_{i}\left(q_{0}\right)$ for $\sigma_{i} \geq 0$. Hence we may regard $U$ as the subspace spanned by the normals $\hat{n}_{i}\left(q_{0}\right)$ for $i=1, \ldots, k$. By definition of $2^{\text {nd }}$ order form-closure, every $\dot{q} \in T_{q_{0}} R^{m}$ which lies in $U$ satisfies $\hat{n}_{i}\left(q_{0}\right) \cdot \dot{q}<0$ for some $1 \leq i \leq k$. Using the same arguments given in the previous lemma, this implies that the vectors $\hat{n}_{1}\left(q_{0}\right), \ldots, \hat{n}_{k}\left(q_{0}\right)$ positively span a neighborhood about the origin in $U$. Next consider tangent vectors $\dot{q}$ in the orthogonal subspace $V$. By definition of $2^{\text {nd }}$ order form closure, the c-space relative curvature is negative definite on $V$. Hence every $\dot{q} \in V$ satisfies $\lambda_{1} \dot{q}^{T} D \hat{n}_{1}\left(q_{0}\right) \dot{q}+\cdots+\lambda_{\mathbf{k}} \dot{q}^{T} D \hat{n}_{k}\left(q_{0}\right) \dot{q}<0$. Since the $\lambda_{i}$ 's are non-negative, every $\dot{q} \in V$ satisfies $\dot{q}^{T} D \hat{n}_{i}\left(q_{0}\right) \dot{q}<0$ for some $1 \leq i \leq k$. Consider now the collection of vectors $\bar{D} \hat{n}_{i}\left(q_{0}\right) \dot{q}$, for all $\dot{q} \in V$ and for $i=1, \ldots, k$. We have just shown that these vectors span the subspace $V$. Otherwise there would exist a vector $\dot{q} \in V$ such that $\dot{q}^{T} D \hat{n}_{i}\left(q_{0}\right) \dot{q}=0$ for $i=1, \ldots, k$. Each $\dot{w}_{i}$ can be realized as $\dot{w}_{i}=\sigma_{i} D \hat{n}_{i}\left(q_{0}\right) \dot{q}$ by instantaneously varying the contact point location while the magnitude $\sigma_{i}$ is held fixed. Hence the collection of all wrenchderivatives, $\dot{w}_{i}$ for $i=1, \ldots, k$, spans the subspace $V$. The arguments given are all reversible and hence the lemma is proved.

\section{Frictional Force and Form Closure}

In this section we briefly consider how to incorporate friction into our mobility theory. We assume the stan- 
dard Coulomb friction model: $\left|F_{1}^{t}\right| \leq \mu\left|F_{i}^{n}\right|$, where $F_{i}^{t}$ and $F_{i}^{n}$ are the tangent and normal components of the $i^{\text {th }}$ finger force, $F_{i}$, and $\mu$ is the coefficient of Coulomb friction.

Let $F C_{i}$ denote the friction cone associated with the $i^{\text {th }}$ finger. Let the $i^{\text {th }}$ finger contact $\mathcal{B}$ at point $x_{i}$ on its boundary. The c-space wrench (c-wrench) due to a single-finger contact force, $F_{i} \in F C_{i}$, on $\mathcal{B}(q)$ is:

$$
w_{i}(q)=\left[D X_{r_{i}}(q)\right]^{T} F_{i},
$$

where $D X_{r_{i}}(q)=\frac{d}{d q} X_{r_{i}}(q)$, and $X_{r_{i}}$ is the rigid body transformation of Eq. (1). The set of forces inside the friction cone maps to a cone of wrenches in c-space. Let $\mathcal{W}_{i}$ represent the $\mathrm{c}$-wrench cone due to the $i^{\text {th }}$ finger: $\mathcal{W}_{i}=\left\{w: w=\left[D X_{r_{i}}\right]^{T} F_{i}, \forall F_{i} \in F C_{i}\right\}$.

In the frictionless case, the set of $1^{\text {st }}$ order free motions was defined using the virtual work principle. That is, $1^{\text {st }}$ order free motions must satisfy $F_{i} \cdot \dot{X}_{r_{i}}=F_{i} \cdot\left[D X_{r_{i}}\right] \dot{q}=\left(\left[D X_{r_{i}}\right]^{T} F_{i}\right) \cdot \dot{q}=w_{i} \cdot \dot{q}>0$, where $F_{i}$ is a force that can be generated by $\mathcal{A}_{i}$.. We can apply this concept here to derive the following.

Definition 6 : Let $\mathcal{B}$ be in frictional contact with stationary rigid finger $\mathcal{A}_{i}$ at configuration $q_{0}$. The set of $1^{\text {st }}$ order unimpeded motions is:

$F M_{i}^{1}\left(q_{0}\right)=\left\{\dot{q} \in T_{q_{0}} \mathbb{R}^{m}: \dot{q} \cdot w_{\alpha} \geq 0 \quad \forall w_{\alpha} \in \mathcal{W}_{i}\right\}$

For $k$ fingers, $F M_{1, \ldots, k}^{1}\left(q_{0}\right)=\cap_{i} F M_{i}^{1}\left(q_{0}\right)$. The set of $1^{\text {st }}$ order impeded motions is:

$$
F I_{i}^{1}=M_{i}^{1}-F M_{i}^{1}
$$

The unimpeded motions are those $1^{\text {st }}$ order motions which are $1^{\text {st }}$ order free, and which can not be affected by frictional forces. For a single contact, the set of unimpeded motions is the cone in $T_{q 0} \mathbb{R}^{m}$ that is polar to the negated wrench cone, $-\mathcal{W}_{i}$. The impeded motions are those motions which are $1^{\text {st }}$ order free, but which can be instantaneously impeded by frictional forces. If $\mathcal{B}$ is contacted by $k$ fingers, then we say it is in frictional equilibrium if there exists $w_{i} \in \mathcal{W}_{i}$ for $i=1, \cdots, k$ such that $\sum_{i=1}^{k} w_{i}=0$. Frictional force and form closure can be now by defined.

Proposition 5.1 Let $\mathcal{B}$ be grasped in a frictional equilibrium by $k$ fingers. Let $\mathcal{W}_{i}$ be the c-wrench cone due to the $i^{\text {th }}$ finger. The grasp is frictional $1^{\text {st }}$ order force closure if there exists a neighborhood about 0 in $\operatorname{co}\left(\mathcal{W}_{i}, \cdots, \mathcal{W}_{k}\right)$, where $c o(\cdot)$ denotes the convex hull. The grasp is $1^{\text {st }}$ order frictional form closure if $\cap_{i=1}^{k} F M_{i}^{1}=\{0\}$.

This definition of frictional force closure is equivalent to its standard definition in the literature. The new frictional form closure definition is dual to the force closure definition.

\section{References}

[1] J. Czyzowicz, I. Stojmenovic, and J. Urrutia. Immobilizing a polytope. In Lecture Notes in Computer Science, volume 519, pages 214-227. 1991.

[2] W. S. Howard and V. Kumar. Modeling and analysis of the compliance and stability of enveloping grasps. In IEEE Int. Conference on Robotics and Automation, pages 1367-1372, Nagoya, Japan, May 1995.

[3] K. Lakshminarayana. Mechanics of form closure. Technical Report 78-DET-32, ASME, 1978.

4] X. Markenscoff, L. Ni, and C. H. Papadimitriou. The geometry of grasping. Int. J. of Robotics Research, 9(1):61-74, Feb 1990.

5] B. Mirtich and J. F. Canny. Optimum force-closure grasps. Technical Report ESRC 93-11/RAMP 93-5, Dept. of EE-CS, U.C. Berkeley, July 1993.

[6] B. Mishra. Workholding. In Proc. IEEE/RSJ International Conference on Intelligent Robots and Sys. tems, pages 53-57, 1991.

[7] V.-D. Nguyen. Constructing force-closure grasps. Int. J. of Robotics Research, 7(3):3-16, June 1988.

[8] M. S. Ohwovoriole and B. Roth. An extension of screw theory. J. of Mechanical Design, 103:725-735, 1981.

[9] F. Reuleaux. The Kinematics of Machinery. Macmillan 1876, republished by Dover, NY, 1963.

[10] E. Rimon and J. W. Burdick. Mobility of bodies in contact: A new $2^{\text {nd }}$ order mobility index for multiplefinger grasps. In Proc. IEEE Int. Conf. on Robotics and Automation, pages 2329-2335, San Diego, CA, May 1994.

[11] E. Rimon and J. W. Burdick. Mobility of bodies in contact: How forces are generated by curvature effects. In IEEE Int. Conf. on Robotics and Automation, pages 2336-2341, San Diego, CA, May 1994.

[12] E. Rimon and J. W. Burdick. A configuration spaće analysis of bodies in contact-part $\mathrm{i}$ : $1^{\text {st }}$ order mobility. Mechanisms and Machine Theory, 30(6):897912, 1995 .

[13] E. Rimon and J. W. Burdick. A configuration space analysis of bodies in contact-part ii: $2^{\text {nd }}$ order mobility. Mechanisms and Machine Theory, 30(6):913$928,1995$.

[14] E. Rimon and J. W. Burdick. New bounds on the number of frictionless fingers required to immobilize planar objects. J. Robotic Systems, 12(6):433-451, June 1995 .

[15] P. Somoff. Uber gebiete von schraubengeschwindigkeiten eines starren korpers bieverschiedener zahl von stuzflachen. Zeitschrift fur Mathematic and Physik, 45:24う-306, 1900.

[16] J. C. Trinkle. A quantitative test for form closure grasps. In Proc. IEEE/RSJ Int. Conf. on Intelligent Robots and Systems, pages 1670-1677, Raleigh, $\mathrm{XC}$, July 1992 .

[17] J. C. Trinkle, A. O. Farahat, and P. F. Stiller. Firstorder stability cells of frictionless rigid body systems. Tech report. CS Dept., Texas A\&M, Jan. 1993.

[18] J. C. Trinkle, A. O. Farahat, and P. F. Stiller. Second-order stability cells of frictionless rigid body systems. In IEEE Int. Conference on Robotics and Automation. San Diego. CA. May 1994. 